\title{
Cornea
}

\section{Tear Cytokine Levels in Contact Lens Wearers with Acanthamoeba Keratitis --Manuscript Draft--}

Manuscript Number:

Full Title:

Article Type:

Keywords:

Corresponding Author:
CORNEA-D-17-00040R2

Tear Cytokine Levels in Contact Lens Wearers with Acanthamoeba Keratitis

Clinical Science

acanthamoeba, keratitis, contact lens, tear, cytokine

Nicole Carnt, BOptom, PhD

Westmead Millennium Institute for Medical Research

Westmead, NSW AUSTRALIA

Corresponding Author Secondary Information:

Corresponding Author's Institution:

Westmead Millennium Institute for Medical Research

Corresponding Author's Secondary Institution:

First Author:

Nicole Carnt, BOptom, PhD

First Author Secondary Information:

Order of Authors:

Nicole Carnt, BOptom, PhD

Vicente Martin Montanez, PhD

Grazyna Galatowicz, BSci

Neyme Veli, BSci

Virginia Calder, $\mathrm{PhD}$

Order of Authors Secondary Information:

Manuscript Region of Origin:

UNITED KINGDOM

Abstract:

\section{ABSTRACT}

Purpose: To determine differences in key tear film cytokines between mild and severe cases of Acanthamoeba Keratitis (AK) and control contact lens (CL) wearers.

Methods: This was a prospective study of CL wearers with AK attending Moorfields Eye Hospital (MEH) and control CL wearers from the Institute of Optometry, London. Basal tear specimens were collected by 10ul capillary tubes (Blaubrand intraMARK, Wertheim, Germany) and tear protein levels were measured with a multiplex magnetic bead array (Luminex 100, Luminex Corporation, Austin, TX) for cytokines IL-1ß, IL-6, IL-8, IL-10, IL-17A, IL-17E, IL-17F, IL-22, and IFNy and with ELISA (Abcam, Cambridge, UK) for CXCL2. Severe cases of AK were defined as having active infection for over 12 months and at least one severe inflammatory event.

Results: One hundred and thirty two tear samples were collected from a total of 61 cases ( 15 severe and 46 mild-moderate) and 22 controls. IL-8, part of the TLR4 cytokine cascade, was found to be expressed at a detectable level more often in cases of AK compared to control CL wearers $(p=0.003)$, and in higher concentrations in severe compared to milder forms of the disease ( $z=-2.35)$. IL-22, part of the IL-10 family, and a proinflammatory Th17 cytokine, was detected more often in severe compared to milder forms of $A K(p<0.02)$.

Conclusion: Profiling Acanthamoeba Keratitis patients during disease shows differences in cytokine levels between severe and milder disease that may inform clinical management. The TLR4 and IL-10/Th17 inflammatory pathways should be included in further investigations of this disease. 
1 Tear Cytokine Levels in Contact Lens Wearers with Acanthamoeba Keratitis

2

3 Authors:

$4 \quad$ Nicole Carnt ${ }^{1,2,3}$ BOptom, PhD

$5 \quad$ Vicente Martin Montenez ${ }^{2} \mathrm{PhD}$

6 Grazyna Galatowicz ${ }^{1}$ BSci

$7 \quad$ Neyme Veli $^{2}$ BSci

$8 \quad$ Virginia Calder ${ }^{1,4} \mathrm{PhD}$

9

$10{ }^{1}$ UCL Institute of Ophthalmology, London, UK

$11{ }^{2}$ Moorfields Eye Hospital NHS Foundation Trust, London, UK

$12{ }^{3}$ University of Sydney, Sydney, Australia

$13{ }^{4}$ National Institute of Health Research (NIHR) Biomedical Research Centre at Moorfields Eye

14 Hospital NHS Foundation Trust \& UCL Institute of Ophthalmology, London, UK

15 Corresponding author:

$16 \quad$ Nicole Carnt

17 Westmead Institute for Medical Research

18176 Hawkesbury Rd

19 Westmead, 2145, 
nicolecarnt@gmail.com

24 Financial Disclosures:

25 Consulting: Nicole Carnt Specsavers Australia and Alcon Laboratories, Inc. Consulting 26 Virginia Calder Allergan plc; Vicente Martin Montenez, Grazyna Galatowicz, Neyme Veli,have 27 no financial disclosures.

29 Key words:

30 acanthamoeba, keratitis, contact lens, tear, cytokine

32 Funding:

33 This study was an Investigator Initiated Study funded by Johnson \& Johnson Vision Care, Inc.

34 Nicole Carnt is supported by and Australian Government National Health and Medical 35 Research Council (NHMRC) CJ Martin Early Career Research Fellowship (APP1036728). The 36 funding for this study, including consultants' time, was supported by the National Institute for 37 Health Research (NIHR) Biomedical Research Centre, based at Moorfields Eye Hospital NHS 38 Foundation Trust and UCL Institute of Ophthalmology. The views expressed are those of the 39 author(s) and not necessarily those of the NHS, the NIHR or the Department of Health. 
Purpose: To determine differences in key tear film cytokines between mild and severe cases of Acanthamoeba Keratitis (AK) and control contact lens $(\mathrm{CL})$ wearers.

44 Methods: This was a prospective study of CL wearers with AK attending Moorfields Eye Hospital $(\mathrm{MEH})$ and control CL wearers from the Institute of Optometry, London. Basal tear specimens were collected by 10ul capillary tubes (Blaubrand intraMARK, Wertheim, Germany) and tear protein levels were measured with a multiplex magnetic bead array (Luminex 100, Luminex Corporation, Austin, TX) for cytokines IL-1ß, IL-6, IL-8, IL-10, IL-17A, IL-17E, IL-17F, IL-22, and IFNy and with ELISA (Abcam, Cambridge, UK) for CXCL2. Severe cases of AK were defined as having active infection for over 12 months and at least one severe inflammatory event.

Results: One hundred and thirty two tear samples were collected from a total of 61 cases (15 severe and 46 mild-moderate) and 22 controls. IL-8, part of the TLR4 cytokine cascade, was found to be expressed at a detectable level more often in cases of AK compared to control CL wearers $(p=0.003)$, and in higher concentrations in severe compared to milder forms of the disease $(\mathrm{z}=-2.35)$. IL-22, part of the IL-10 family, and a proinflammatory Th17 cytokine, was detected more often in severe compared to milder forms of $A K(p<0.02)$.

Conclusion: Profiling Acanthamoeba Keratitis patients during disease shows differences in cytokine levels between severe and milder disease that may inform clinical management. The TLR4 and IL-10/Th17 inflammatory pathways should be included in further investigations of this disease.

62 
64 Acanthamoeba Keratitis (AK) is one of the most severe forms of corneal infection, with over $90 \%$ of cases occurring in contact lens (CL) wearers. ${ }^{1}$ Vision loss occurs in $33 \%$ of patients, with corneal transplantation required in around $26 \% .{ }^{2}$ Recent reports, and case monitoring at our centre, show that the numbers of AK cases are increasing. ${ }^{3,4}$ AK generally affects a young and otherwise healthy group of individuals ${ }^{5}$ in whom lifetime disability costs are high. As well as the long term effects, such as decreased quality of life, and loss of productivity due to reduced vision, there are significant short term costs to sufferers and carers, such as loss of wages and distress, in addition to symptoms such as severe pain and light sensitivity experienced by sufferers. ${ }^{6}$

Some complications associated with CLs are somewhat controlled by the release of tear inflammatory molecules, such as giant papillary conjunctivitis which is characterized by altered levels of eotaxin ${ }^{7}$ or corneal neovascularization which is mediated by vascular endothelial growth factor (VEGF). ${ }^{8}$ Moreover, it has been shown that CL wearers with CL-induced acute red eye present higher concentrations of IL-8 than healthy subjects ${ }^{9}$. Others ${ }^{10-13}$ have indicated altered levels of tear cytokines such as interleukin (IL)-6, IL-8 and epidermal growth factor (EGF) during CL wear. However, to this day little is known about the tear inflammatory mediation in AK. Profiling AK patients during disease could show differences in cytokine levels between severe and milder disease that may inform clinical management.

The aim of this study is to determine the differences in cytokine levels in CL-wearing patients with AK compared to $\mathrm{CL}$ wearers without the disease. A secondary goal is to investigate differences in cytokine levels between patients with severe forms of AK and those with mildmoderate forms of this infection 
87 This was a prospective case control study of CL wearers with AK attending Moorfields Eye

88 Hospital (MEH) and control CL wearers from the Institute of Optometry, London. The research

89 protocol adhered to the tenets of the Declaration of Helsinki and was approved by the local

90 ethics committee. Written informed consent was obtained from all participants.

\section{Participants}

92

Severe cases of AK were defined as having active infection for over 12 months and having had at least one severe inflammatory event such as scleritis, persistent non-healing defect (for 14 days or more) and/or pupil paralysis. Mild-moderate cases had recurrent disease in the absence of severe inflammatory events or disease that required active treatment for less than and up to 12 months.

\section{Tear sample collection}

Samples were collected from AK patients at follow-up visits during their treatment on a convenience basis depending on the flow of the clinic visits. Collection times varied between 10am and $4 \mathrm{pm}$. Samples were collected from the affected eye only. For bilateral cases, the worst affected eye was sampled.

Samples were collected from control CL wearers at the conclusion of routine aftercare appointments at the Institute of Optometry. Lenses may or may not have been worn according to the patient preference at the end of the appointment. So as to not affect the equilibrated tear milieu, that status remained for tear collection. For these control CL wearers, samples were collected from the right eye, and switched to the left should no sample be obtained from the right eye. In monocular wearers, the eye sampled was the CL wearing eye.

Tear samples for both AK cases and controls were basal tear specimens collected by 10ul capillary tubes (Blaubrand intraMARK, Wertheim, Germany) and stored in ethylenediaminetetraacetic acid (EDTA) coated 0.5ml Eppendorf tubes. Following collection, 
111 the samples were kept cold using a standard cool box and ice packs. Upon delivery to the

112 laboratory on the same day, the samples were centrifuged at 1,600rpm for 5 minutes. The

113 cell-free supernatant was then pipetted into clean EDTA coated 0.5ml Eppendorf tubes and

114 stored at $-80^{\circ} \mathrm{C}$ prior to analysis.

115 Analysis of tear molecules

116 Cytokines IL-1 $\beta$, IL-6, IL-8, IL-10, IL-17A, IL-17E, IL-17F, IL-22, interferon (IFNy) and

117 chemokine (C-X-C motif) ligand 2 (CXCL2) were chosen for analysis based on established

118 and hypothesised inflammatory pathways in AK. Tear protein levels were measured with a

119 multiplex bead array using the Luminex based platform (Luminex 100, Luminex Corporation,

120 Austin, TX) for all analytes apart from CXCL2. CXCL2 was measured with an enzyme-linked

121 immunosorbent assay (ELISA, Abcam, Cambridge, UK) as this protein was not compatible

122 with the chosen Luminex range of targets.

123 Samples were diluted with the respective kit reagent depending on the sample volume and

124 normalised for analysis. Standard curves using duplicate known dilutions were generated for 125 the Luminex and ELISA analysis. Luminex data were analysed with the instrument software 126 and raw scores of the ELISA optical density were converted to concentrations in Excel 2010 127 (Microsoft). Concentrations lower than the detectable limits were labelled as not detectable 128 (ND). Final concentrations above the minimum detectable limit were adjusted for the dilution 129 factor.

130 Data analysis

131 Statistics were analysed using Graphpad.com/online calculator and Microsoft Excel 2010 132 software.

133 Differences between cases and controls and between severe and mild-moderate cases were determined as follow: Fishers exact test was performed to determine the proportions of 
135 detectable samples and Mann-Whitney $U$ test was used for the sample quantities over the

136 detectable levels.

$137 \quad P$ values less than or equal to 0.05 were considered statistically significant.

RESULTS

139 One hundred and thirty two tear samples were collected from a total of 61 AK cases ( 15 severe 140 and 46 mild-moderate) and 22 controls. There were no differences in gender distribution 141 between case and control groups $(p=0.06)$, however significant differences in age were found 142 between the groups $(p<0.001)$. In addition, there were more daily disposable wearers in the 143 control group compared to the AK cases $(p=0.02)$. Descriptive data detailing age, gender and 144 lens type, are shown in Table 1.

145 Levels of IL-6, IL-8, IL-22 and IL-17E were readily detectable. The levels of IFNy, IL-17F, IL$14617 \mathrm{~A}$, IL-10, IL-27 and IL-1 $\beta$ were below the minimum detectable limit for all case and control 147 samples. The proportion of non detectable (ND) samples for each protein are detailed in Table 1482.

149 Cases vs. controls

150 Figure 1 shows the proportion of cytokines for the cases and controls for each of the molecules

151 for which there was more than 1 positive sample (IL-1 $\beta$ was detectable in only one sample, 152 and was considered "non detectable" for this study). There were more samples with detectable 153 levels of IL-8 in the cases compared to the controls $(p=0.003)$. Almost half of the tear 154 specimens in both groups had detectable levels of IL-22, whereas IL-6 and IL-17E showed 155 very low frequencies of positivity. The one control with a positive sample for IL-6 was not the

156 same control that was the only control sample positive for IL-17E. There was no difference

157 between the CXCL2 levels for cases and controls with more than $75 \%$ of tear specimens 158 yielding detectable quantities of this molecule (cases 56/67, 84\% and controls, 10/13, 77\%). 
159 Figures 2-5 show the concentrations of IL-6, IL-8, IL-22, and IL-17E, respectively in tears of

160 individual cases (by visit) and individual controls that measured above detectable limits by

161 Luminex. Figure 6 shows the concentrations CXCL2 in tears of individual cases (by visit) and 162 individual controls that measured above detectable limits by ELISA. There was no difference 163 between the median concentration of IL-8, IL-22 and CXCL2 in tears of cases and controls 164 ( $\mathrm{z}=-0.57, \mathrm{z}=0.97$ and $\mathrm{Z}=0.05$ respectively). Only one control sample was positive for IL-6 and 165 IL-17E and so Mann-Whitney U Test could not be performed.

166 Severe vs. mild-moderate cases

167 Figure 7 shows the proportions of detectable protein samples (IL-8, IL-22, IL-6 and IL-17E) 168 investigated with Luminex for severe compared to mild-moderate cases. IL-22 was less likely 169 to be detected amongst the mild-moderate cases compared to the severe cases of AK $170(p=0.02)$, however there was no difference between mild-moderate cases and severe cases 171 for the proteins, IL-8, IL-6 and IL-17E ( $p=0.48, p=0.27$ and $p=1.0$ respectively). There was also 172 no difference in CXCL2 levels between the severe and moderate/mild cases (23/29, 79.3\% 173 compared to $33 / 38,86.8 \%, p=0.41$ )

174 Table 3 shows the median tear protein concentrations for severe compared to the mild175 moderate samples. There was a higher level of IL-8 detectable in the tears of severe cases compared to the mild-moderate cases of this infection $(z=-2.31)$, however there was no

177 difference between tear protein levels of IL-22, IL-6, IL-17E and CXCL2).

179 The present study was the first to examine the cytokine levels in patients with mild compared 180 to more severe AK, and compare these to control CL wearers. This study has highlighted IL1818 as a key molecule in the AK inflammatory response, and there is also some evidence for 182 cell mediated inflammatory response involving the IL-17 pathway, via IL-22. 
183 IL-8 was found to be expressed at a detectable level measured by Luminex more often in

184 cases of AK compared to control CL wearers, and in higher concentrations in more severe 185 compared to milder forms of the disease. IL-8 is a key inflammatory chemokine that mobilises 186 and activates neutrophils. ${ }^{7}$ Neutrophils are essential components of the early inflammatory 187 response to Acanthamoeba. ${ }^{8}$ Furthermore, IL-8 is part of the toll like receptor 4 (TLR-4) 188 cascade which initiates the cytokine response in $\mathrm{AK} .{ }^{9} \mathrm{IL}-8$ also promotes angiogenesis in the 189 eye $^{7}$ and further characterisation of patients that develop neovascularisation in AK may reveal 190 differences in levels that may predict patients who go on to develop this complication, and 191 more targeted management such as frequent topical steroids may be advocated in these 192 cases. Neovascularisation is a contraindicated in corneal transplant candidates, often the last 193 resort to significantly improve vision in AK patients. Keratoplasty is required for visual 194 rehabilitation in around $12 \%$ of $\mathrm{AK}_{\text {cases. }}^{2}$

IL-22, part of the IL-10 family, and a proinflammatory Th17 cytokine, ${ }^{10}$ was detected more often in severe compared to milder forms of AK. IL-22 may prolong the inflammatory response and, in severe forms of disease, this may be beneficial to control infection but may also be involved in tissue destruction due to inflammation.

Most of the IL-17 cytokines were not detected in levels high enough to be measured in the tears in these subjects using Luminex technology. Since multiplex bead arrays are well established as being one of the more sensitive methods of detection for low levels of analytes, the specimens with no detectable levels were presumed negative. It may be useful to compare the IL-17E cytokine, which was expressed by a small number of cases and one control, using 204 ELISA, in another cohort of samples. Like IL-22, IL-17 has been implicated in chronic 205 inflammatory conditions ${ }^{11}$ and IL-17A has recently been shown to be protective against Acanthamoeba keratitis severity in a mouse model. ${ }^{12}$ This contrasts with keratitis caused by

207 Herpes Simplex Virus and Pseudomonas where IL-17A is associated with an increased 208 corneal inflammatory response. ${ }^{13-15}$. IL-17A is known as a "double sword" agent; in some 
209 circumstances it protects the host and in others, it results in chronic inflammation and tissue 210 damage. ${ }^{16}$ ) IL-17A both initiates and activates neutrophils and is also produced by 211 neutrophils. ${ }^{17}$ Recently, a novel population of neutrophils were characterized, that are capable 212 of autocrine IL-17A activity, which leads to increased death of fungal hyphae in a murine model 213 of Aspergillus corneal infection. ${ }^{18}$

214 CXCL2 (also known as macrophage inflammatory protein 2-alpha, MIP2- $\alpha$ ) appears to be 215 constitutively expressed in AK cases and control CL wearers and not up- or down-regulated 216 in this disease. MIP2 has been shown to be important in animal models of AK. ${ }^{19}$ Animal models 217 of disease do not exhibit the severe inflammatory complications of AK, such as scleritis ${ }^{5}$, and 218 inflammatory pathways may vary somewhat between humans and animal models.

219 IL-6, a proinflammatory cytokine with several functions, was only detected in one control 220 sample; either this study did not have enough power to show differences between cases and 221 controls or IL-6 is not important in the inflammatory response in this disease. Furthermore it is 222 possible that there is a defect in IL-6 at the protein level. Our group has found that single 223 nucleotide polymorphisms (SNP) of IL-6 genes are implicated in the susceptibility and severity of bacterial keratitis in CL wearers. ${ }^{20} \mathrm{IL}-6$ is a key player in the IL-22 and IL-17 pathways ${ }^{11}$ and it would be prudent to further investigate this protein as a candidate in future immunological analysis in AK.

Cytokine and chemokine profiles correlate with several inflammatory anterior eye disease states such as dry eye, ${ }^{21-24}$ allergic eye disease, ${ }^{25,} 26$ the autoimmune condition, Sjogren's syndrome, ${ }^{27-29}$ vernal keratoconjunctivitis ${ }^{30}$ and ocular rosacea. ${ }^{31}$ Two studies have highlighted tear protein profiles associated with bacterial ${ }^{32}$ and fungal keratitis. ${ }^{33}$

231 In bacterial keratitis, cytokines and chemokines are upregulated in both the affected and 232 contralateral eye, and these changes have been correlated with cellular changes imaged on 233 the ocular surface ${ }^{32}$. Specifically, IL-1, IL-6 and IL-8 were elevated in the 'infected' tears 
compared to non-affected controls. Changes were also found in the contralateral eye of 235 bacterial keratitis patients, namely the upregulation of chemokine ligand 2 (CCL-2), IL-10 and IL-17a. TREM-1 was also elevated in both the affected and contralateral eyes. Changes in tear cytokines were correlated with dendritic cell and sub-basal nerve fibre presence and morphology, as follows; tear concentrations of the proinflammatory cytokines, IL-1B, IL-6, IL8 and IL-17a were positively correlated with dendritic cell density, and IL-1B, IL-6, IL-8 and TREM-1 were inversely correlated with sub-basal nerve density.

Proteomic analyses have been used in an Indian study of fungal keratitis patients compared to controls to examine differences between tear proteins. Seven protein levels varied between the cases and controls: Prolactin inducible protein and serum albumin precursor were up regulated in the infected samples; Cystatin S precursor, cystatin SN precursor, cystatin, and human tear lipocalin were downregulated in the infected samples; glutaredoxin-related protein was found only in the infected samples ${ }^{33}$.

247 Concentrations of the following cytokines for all subjects in this study fell below the detectable 248 limit for IFNy, IL-10, IL-1 $\beta$, IL-27 as well as IL-17F and IL-17A. Cross reactivity of the antibodies and/or poor sensitivity of the array are unlikely to be implicated since bead-based Luminex technology is one of the most sensitive assays available and has successfully allowed detection of cytokines in tear fluids. ${ }^{34}$ It is possible that these cytokines were masked from detection in the tear specimens due to a build-up of protein and debris at the ocular surface. Alternatively, these cytokines might not be involved in this disease but, until a larger cohort of specimens and controls is investigated, this cannot be assumed.

The differences in cytokine levels found in this study may be due to the effects of the disease on the immune system and/or due to differences in the individual's immune profile at the gene level. Being such a rare disease, it is impossible to conduct a prospective study and compare cytokine levels before and during AK disease, however future studies that assess variations in the DNA structure of these genes in patients will provide more insight into this conundrum. 
260 Furthermore the differences between mild/moderate and severe disease may be due to 261 differences in strains of Acanthamoeba organism. The majority of Acanthamoeba spp that 262 cause keratitis are from the T4 group based on 18s RNA genotyping that separates strains 263 into 17 evolutionary clades or groups (T1-T17). Preliminary information from one study indicates that strains with non-T4 genotypes may cause more severe disease, ${ }^{35}$ however, only three cases of non-T4 AK were compared to 14 T4 genotypes and confirmation in a larger study is required. As genetic profiling of Acanthamoeba spp. allows more refined typing, as can be seen by the mitochondrial cytochrome oxidase (Cox) gene sequencing, ${ }^{36}$ and greater number of cases are reported from other $\mathrm{T}$ strains ${ }^{37,38}$ correlation between different strains and the outcomes of AK may be found. Human biomarker profiling alongside in vitro and animal models will be key to future understanding of the interplay between the host immune system and organism virulence that is evidenced in some conditions such as malaria. ${ }^{39}$

272 A limitation of the present study could be that AK cases were younger than controls. Tear 273 investigations have generally been limited to normals or certain conditions affecting specific 274 age groups and differences between normals across a range of ages has not been shown. 275 Dry eye is more prevalent in older individuals, and increased levels of two cytokines measured 276 in this study, IL-6 and IL-8 have been found in elevated levels in dry eye patients. ${ }^{21-24}$ The 277 controls in this study, although older than the cases, were successful CL wearers, and are 278 unlikely to have had significant dry eye disease. In any case, had some of the cases been on 279 the dry eye spectrum, this would have only potentially masked greater differences in IL-8 levels 280 and would not have affected the IL-6 results, in which only one control showed a reading 281 above the detectable level.

282 More daily disposable wearers were in the control group compared to AK patients in this study.

283 This likely reflects the evidence that AK is more often a disease that occurs in reusable lens 284 wearers, as the environmental contamination of lens cases supports the growth of 285 Acanthamoeba spp. ${ }^{40}$ Only one study has evaluated the tear profile while wearing different 
lens types; using lotrafilcon B (O2OPTIX; CIBA VISION, Duluth, Atlanta, GA) or senofilcon A

287 (Acuvue Oasys; Johnson \& Johnson Vision Care, Inc., Jacksonville, FL), no differences in 288 levels on matrix metalloproteinase 9 (MMP-9), tissue inhibitor of metalloproteinases 1 (TIMP289 1) and neutrophil gelatinase-associated lipocalin (NGAL) during adapted daily wear were 290 found. ${ }^{41}$ It is unlikely that even if lens wear type had an effect on tear cytokine/chemokine 291 levels that this would confound results in the present study as all the AK patients and a proportion of the controls were not wearing lenses at the time of collection.

Another limitation of the study might be the time of the tear samples collection. The tear collection time was scheduled between $10 \mathrm{am}$ and $4 \mathrm{pm}$ to minimise possible diurnal effect and disruption to the $\mathrm{MEH}$ and $\mathrm{IO}$ clinics. While there are recent publications showing a diurnal change of certain tear cytokines and chemokines they indicate a difference between daytime and evening intervals (11am-1pm vs $5 \mathrm{pm}-7 \mathrm{pm})^{42}$; 12am (midday) compared to $9-12 \mathrm{pm}$ (midnight)..$^{43}$ It is improbable that there would be a major variation in cytokine and chemokine levels during the 6-hour daytime interval in which we sampled tears.

This study highlights key areas for future investigation of the pathogenesis of AK. We have shown that in a clinical setting, we can collect tears from patients with AK that may indicate the inflammatory status of the eye. Further investigation of cytokines not detected in this study, and other candidates in the pathways indicated by this analysis, may define a wider spectrum of cytokine changes. In association with careful tracking of patients during the disease process, we may be able to predict when the inflammatory status is changing. This information may help the clinician to better understand the clinical picture and make more informed decisions on individual AK patient management. 
310 We would like to thank the following people for their contribution to the study: Patients with 311 Acanthamoeba Keratitis and control contact lens wearers who donated their tears, Prof John

312 Dart and the External Disease service at Moorfields Eye Hospital, Ms Judith Morris and 313 Institute of Optometry for the recruitment of controls, and Ms Sophie Connor at Moorfields Eye 314 Hospital who helped coordinate the study.

315

316 REFERENCES

317

318 
320 Figure 1. The distribution of the detectable samples for each analyte tested with Luminex for

$321 \quad$ AK case samples and controls

322 Figure 2. IL-6 protein levels above minimum detectable for individual cases (by visit) and

323 individual controls measured by Luminex

324 Figure 3. IL-8 protein levels above minimum detectable for individual cases (by visit) and 325 individual controls measured by Luminex

326 Figure 4. IL-22 protein levels above minimum detectable for individual cases (by visit) and 327 individual controlsas measured by Luminex

328 Figure 5. IL-17E protein levels above minimum detectable for individual cases (by visit) and 329 individual controlsas measured by Luminex

330 Figure 6 CXCL2 protein levels above minimum detectable for individual cases (by visit) and 331 individual controls measured by ELISA

332 Figure 7. Detectable sample distribution for severe compared to mild-moderate AK cases 333 measured with Luminex (mod=moderate)

1. Niederkorn JY, Alizadeh $\mathrm{H}$, Leher $\mathrm{H}$, et al. The pathogenesis of Acanthamoeba keratitis. Microbes and infection / Institut Pasteur. 1999;1:437-443.

2. Robaei D, Carnt N, Minassian DC, et al. Therapeutic and optical keratoplasty in the management of Acanthamoeba keratitis: risk factors, outcomes, and summary of the literature. Ophthalmology. 2015;122:17-24.

3. Yoder JS, Verani J, Heidman N, et al. Acanthamoeba keratitis: the persistence of cases following a multistate outbreak. Ophthalmic Epidemiol. 2012;19:221-225. 
4. Jasim H, Knox-Cartwright $\mathrm{N}$, Cook $\mathrm{S}$, et al. Increase in acanthamoeba keratitis may be associated with use of multipurpose contact lens solution. BMJ. 2012;344:e1246.

5. Dart JK, Saw VP, Kilvington S. Acanthamoeba keratitis: diagnosis and treatment update 2009. Am J Ophthalmol. 2009;148:487-499 e482.

6. Keay L, Edwards K, Naduvilath T, et al. Factors affecting the morbidity of contact lens related microbial keratitis: a population study. Invest Ophthalmol Visual Sci. 2006;47:4302-4308.

7. Ghasemi H, Ghazanfari T, Yaraee R, et al. Roles of IL-8 in Ocular Inflammations: A Review. Ocul Immunol Inflamm. 2011;19:401-412.

8. Clarke DW, Niederkorn JY. The immunobiology of Acanthamoeba keratitis. Microbes and infection / Institut Pasteur. 2006;8:1400-1405.

9. Hoti SL, Tandon V. Ocular parasitoses and their immunology. Ocul Immunol Inflamm. 2011;19:385-396.

10. Dudakov JA, Hanash AM, van den Brink MR. Interleukin-22: immunobiology and pathology. Annu Rev Immunol. 2015;33:747-785.

11. Sabat R, Witte E, Witte K, et al. IL-22 and IL-17: An Overview. In: Quesniaux V, Ryffel, B, Padova F, eds. IL-17, IL-22 and Their Producing Cells: Role in Inflammation and Autoimmunity. Basel: Springer; 2013.

12. Suryawanshi A, Cao Z, Sampson JF, et al. IL-17A-mediated protection against Acanthamoeba keratitis. J Immunol. 2015;194:650-663.

13. Suryawanshi A, Veiga-Parga T, Rajasagi NK, et al. Role of IL-17 and Th17 cells in herpes simplex virus-induced corneal immunopathology. J Immunol. 2011;187:19191930.

14. Suryawanshi A, Veiga-Parga T, Reddy PB, et al. IL-17A differentially regulates corneal vascular endothelial growth factor (VEGF)-A and soluble VEGF receptor 1 expression and promotes corneal angiogenesis after herpes simplex virus infection. $J$ Immunol. 2012;188:3434-3446.

15. Suryawanshi A, Cao Z, Thitiprasert T, et al. Galectin-1-mediated suppression of Pseudomonas aeruginosa-induced corneal immunopathology. $J$ Immunol. 2013;190:6397-6409.

16. Hemdan NY, Abu El-Saad AM, Sack U. The role of Thelper (TH) 17 cells as a doubleedged sword in the interplay of infection and autoimmunity with a focus on xenobioticinduced immunomodulation. Clin Dev Immunol. 2013;2013:374769.

17. Taylor PR, Pearlman E. IL-17A production by neutrophils. Immunol Lett. 2016;169:104-105.

18. Taylor PR, Roy S, Leal SM, Jr., et al. Activation of neutrophils by autocrine IL-17A-IL$17 R C$ interactions during fungal infection is regulated by IL-6, IL-23, RORgammat and dectin-2. Nat Immunol. 2014;15:143-151. 
19. Hurt M, Apte $\mathrm{S}$, Leher $\mathrm{H}$, et al. Exacerbation of Acanthamoeba keratitis in animals treated with anti-macrophage inflammatory protein 2 or antineutrophil antibodies. Infect Immun. 2001;69:2988-2995.

20. Carnt NA, Willcox MD, Hau S, et al. Association of single nucleotide polymorphisms of interleukins-1beta, -6 , and $-12 \mathrm{~B}$ with contact lens keratitis susceptibility and severity. Ophthalmology. 2012;119:1320-1327.

21. Massingale ML, Li X, Vallabhajosyula M, et al. Analysis of inflammatory cytokines in the tears of dry eye patients. Cornea. 2009;28:1023-1027.

22. Lam H, Bleiden L, de Paiva CS, et al. Tear cytokine profiles in dysfunctional tear syndrome. Am J Ophthalmol. 2009;147:198-205 e191.

23. Boehm N, Riechardt Al, Wiegand M, et al. Proinflammatory cytokine profiling of tears from dry eye patients by means of antibody microarrays. Invest Ophthalmol Vis Sci. 2011;52:7725-7730.

24. Enriquez-de-Salamanca A, Castellanos E, Stern ME, et al. Tear cytokine and chemokine analysis and clinical correlations in evaporative-type dry eye disease. $\mathrm{Mol}$ Vis. 2010;16:862-873.

25. Leonardi A, Curnow SJ, Zhan H, et al. Multiple cytokines in human tear specimens in seasonal and chronic allergic eye disease and in conjunctival fibroblast cultures. Clin Exp Allergy. 2006;36:777-784.

26. Cook EB, Stahl JL, Lowe L, et al. Simultaneous measurement of six cytokines in a single sample of human tears using microparticle-based flow cytometry: allergics vs. non-allergics. J Immunol Methods. 2001;254:109-118.

27. Jones DT, Monroy D, Ji Z, et al. Sjogren's syndrome: cytokine and Epstein-Barr viral gene expression within the conjunctival epithelium. Invest Ophthalmol Vis Sci. 1994;35:3493-3504.

28. Pflugfelder SC, Jones D, Ji Z, et al. Altered cytokine balance in the tear fluid and conjunctiva of patients with Sjogren's syndrome keratoconjunctivitis sicca. Curr Eye Res. 1999;19:201-211.

29. Tishler M, Yaron I, Geyer O, et al. Elevated tear interleukin-6 levels in patients with Sjogren syndrome. Ophthalmology. 1998;105:2327-2329.

30. Shoji J, Inada N, Sawa M. Antibody array-generated cytokine profiles of tears of patients with vernal keratoconjunctivitis or giant papillary conjunctivitis. Jpn $J$ Ophthalmol. 2006;50:195-204.

31. Barton K, Monroy DC, Nava A, et al. Inflammatory cytokines in the tears of patients with ocular rosacea. Ophthalmology. 1997;104:1868-1874.

32. Yamaguchi T, Calvacanti BM, Cruzat A, et al. Correlation between human tear cytokine levels and cellular corneal changes in patients with bacterial keratitis by in vivo confocal microscopy. Invest Ophthalmol Vis Sci. 2014;55:7457-7466.

33. Ananthi S, Chitra T, Bini R, et al. Comparative analysis of the tear protein profile in mycotic keratitis patients. Mol Vis. 2008;14:500-507. 
34. Hagan S, Tomlinson A. Tear fluid biomarker profiling: a review of multiplex bead analysis. Ocul Surf. 2013;11:219-235.

35. Arnalich-Montiel F, Lumbreras-Fernandez B, Martin-Navarro CM, et al. Influence of Acanthamoeba genotype on clinical course and outcomes for patients with Acanthamoeba keratitis in Spain. J Clin Microbiol. 2014;52:1213-1216.

36. Kilvington S, Gray T, Dart J, et al. Acanthamoeba keratitis: the role of domestic tap water contamination in the United Kingdom. Invest Ophthalmol Vis Sci. 2004;45:165169.

37. Walochnik J, Scheikl U, Haller-Schober EM. Twenty years of acanthamoeba diagnostics in Austria. J Eukaryot Microbiol. 2015;62:3-11.

38. Grun AL, Stemplewitz B, Scheid P. First report of an Acanthamoeba genotype T13 isolate as etiological agent of a keratitis in humans. Parasitol Res. 2014;113:23952400.

39. Preiser $\mathrm{P}$, Kaviratne $\mathrm{M}$, Khan $\mathrm{S}$, et al. The apical organelles of malaria merozoites: host cell selection, invasion, host immunity and immune evasion. Microbes Infect. 2000;2:1461-1477.

40. Larkin DF, Kilvington S, Easty DL. Contamination of contact lens storage cases by Acanthamoeba and bacteria. Br J Ophthalmol. 1990;74:133-135.

41. Markoulli M, Papas E, Cole N, et al. Effect of contact lens wear on the diurnal profile of matrix metalloproteinase 9 in tears. Optom Vis Sci. 2013;90:419-429.

42. Benito MJ, Gonzalez-Garcia MJ, Teson M, et al. Intra- and inter-day variation of cytokines and chemokines in tears of healthy subjects. Exp Eye Res. 2014;120:43-49.

43. Uchino E, Sonoda S, Kinukawa N, et al. Alteration pattern of tear cytokines during the course of a day: diurnal rhythm analyzed by multicytokine assay. Cytokine. 2006;33:36-40. 
Table 1. Descriptive data of participants recruited for the study.

\begin{tabular}{|c|c|c|c|}
\hline 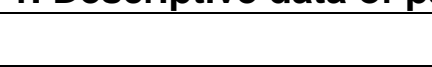 & AK Cases $(n=61)$ & Controls $(n=22)$ & $p$ value \\
\hline Age, years, mean (SD) & $35.4 \pm 13.6$ & $52.7 \pm 15.4$ & $<0.001$ \\
\hline $\begin{array}{l}\text { Gender, n (\%) } \\
\text { Males } \\
\text { Females } \\
\end{array}$ & $\begin{array}{l}28(45.9) \\
33(54.1) \\
\end{array}$ & $\begin{array}{c}5(22.7) \\
17(77.3) \\
\end{array}$ & 0.06 \\
\hline $\begin{array}{l}\text { Type of CL worn, } \mathbf{n} \text { (\% } \\
\text { known) } \\
\text { Daily soft } \\
2-4 \text { weeks disposable soft } \\
>1 \text { month replacement soft } \\
\text { unknown }\end{array}$ & $\begin{array}{c}9(20.0) \\
33(73.3) \\
3(6.7) \\
16\end{array}$ & $\begin{array}{c}14(63.6) \\
7(31.8) \\
1(4.5) \\
0\end{array}$ & 0.02 \\
\hline
\end{tabular}

$\mathrm{SD}=$ standard deviation; $\mathrm{CL}=$ contact lens 
Table 2. Proportion of non detectable samples for cases and controls.

\begin{tabular}{l|c|c}
\hline & AK Cases & Controls \\
\hline ND Analyte, $\mathbf{n}(\%)$ & $104 / 120(86.7)$ & $10 / 11(90.9)$ \\
IL-6 & $24 / 120(20.0)$ & $7 / 11(63.6)$ \\
IL-8 & $74 / 120(61.7)$ & $7 / 11(63.6)$ \\
IL-22 & $114 / 120(95.0)$ & $10 / 11(90.9)$ \\
IL-17E & $11 / 69(15.9)$ & $3 / 11(27.3)$ \\
CXCL2 & $119 / 120(99.2)$ & $11 / 11(100)$ \\
IL-1 $\beta$ & $120 / 120(100)$ & $11 / 11(100)$ \\
IFNy & $120 / 120(100)$ & $11 / 11(100)$ \\
II-17F & $120 / 120(100)$ & $11 / 11(100)$ \\
IL-17A & $120 / 120(100)$ & $11 / 11(100)$ \\
IL-10 & $120 / 120(100)$ & $11 / 11(100)$ \\
IL-27 & & \\
\hline
\end{tabular}

$\mathrm{ND}=$ non detectable 
Table 3. Median concentrations and 95\% confidence intervals $(\mathrm{Cl})$ for cytokines in tear samples of severe compared to mild-moderate cases.

\begin{tabular}{|c|c|c|c|c|c|c|c|}
\hline \multirow{2}{*}{ Cytokine } & \multicolumn{3}{|c|}{ Severe } & \multicolumn{3}{|c|}{ Mild-Moderate } & \multirow{2}{*}{$Z$ value } \\
\hline & $\mathbf{n}$ & median & $95 \% \mathrm{Cl}$ & $\mathbf{n}$ & median & $95 \% \mathrm{Cl}$ & \\
\hline IL-8 & 36 & 162.4 & $72.8-447.3$ & 60 & 66.2 & $57.6-119.5$ & -2.31 \\
\hline IL-22 & 22 & 470.8 & $313.5-1237.0$ & 24 & 671.6 & 214.9-1501.0 & -0.44 \\
\hline IL-6 & 9 & 145.0 & $31.9-1361.5$ & 7 & 80.9 & $16.8-391.0$ & 1.27 \\
\hline IL-17E & 2 & 7265.1 & $\mathrm{~N} / \mathrm{A}$ & 4 & 2587.1 & $\mathrm{~N} / \mathrm{A}$ & -0.93 \\
\hline CXCL2 & 22 & 3173.3 & $1150.9-4110.7$ & 34 & 3007.2 & 1847.5-3703.9 & -0.23 \\
\hline
\end{tabular}

n: number of samples; $\mathrm{Cl}$ : confidence index; N/A: not applicable 


\section{Luminex cases vs control detectable samples}

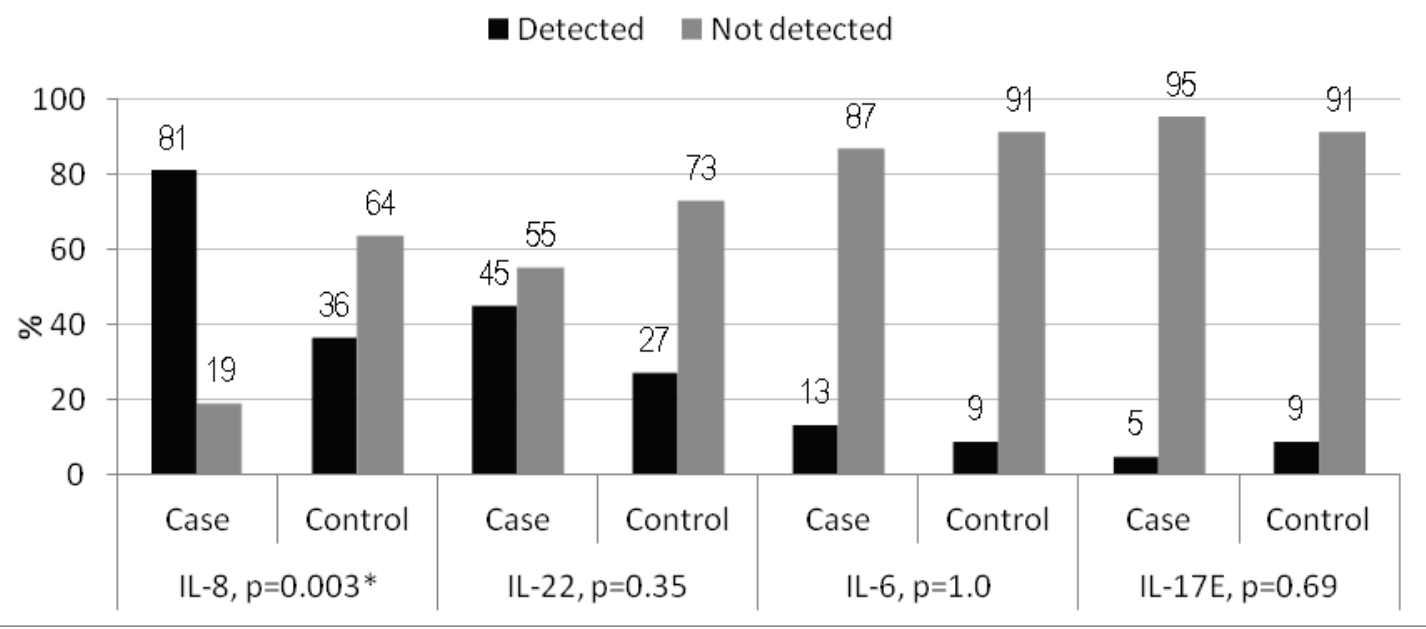




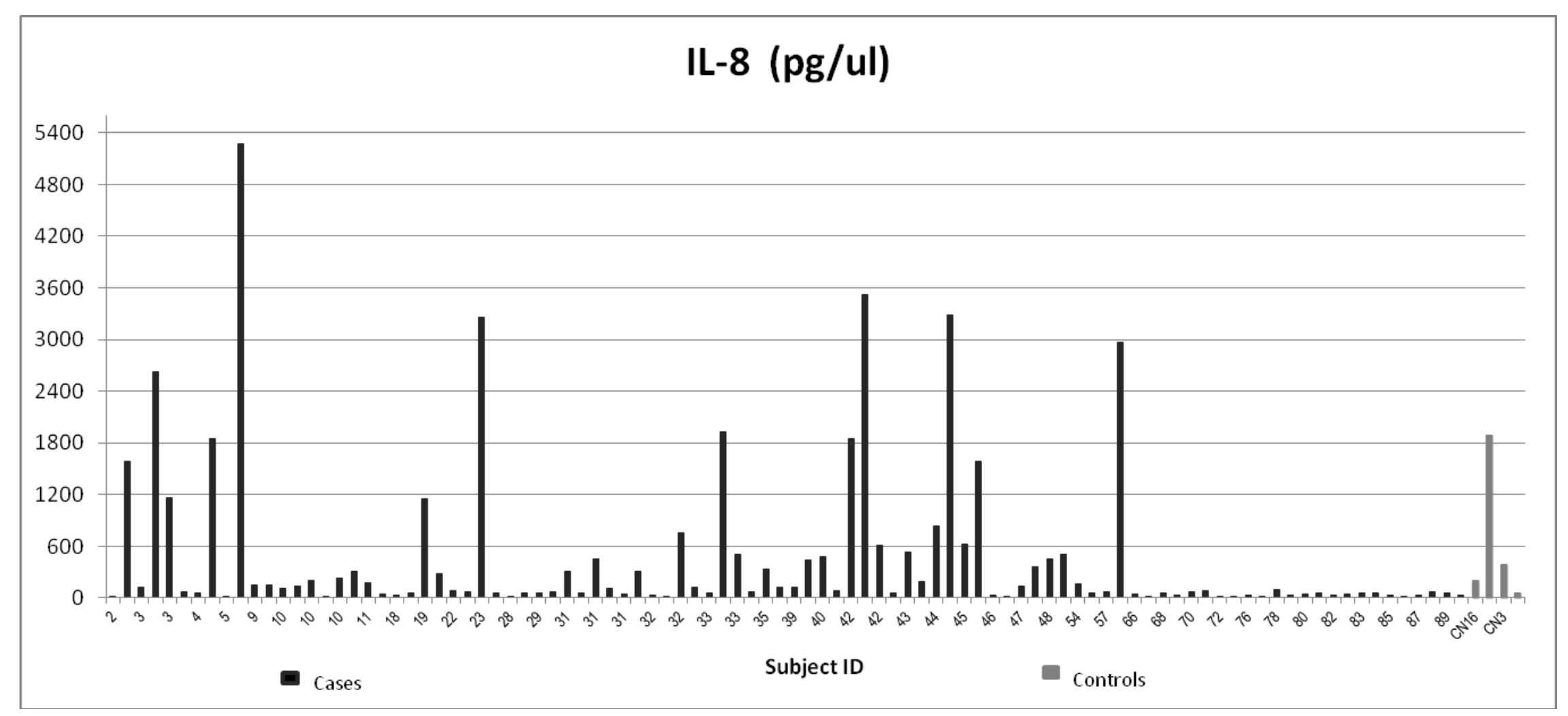




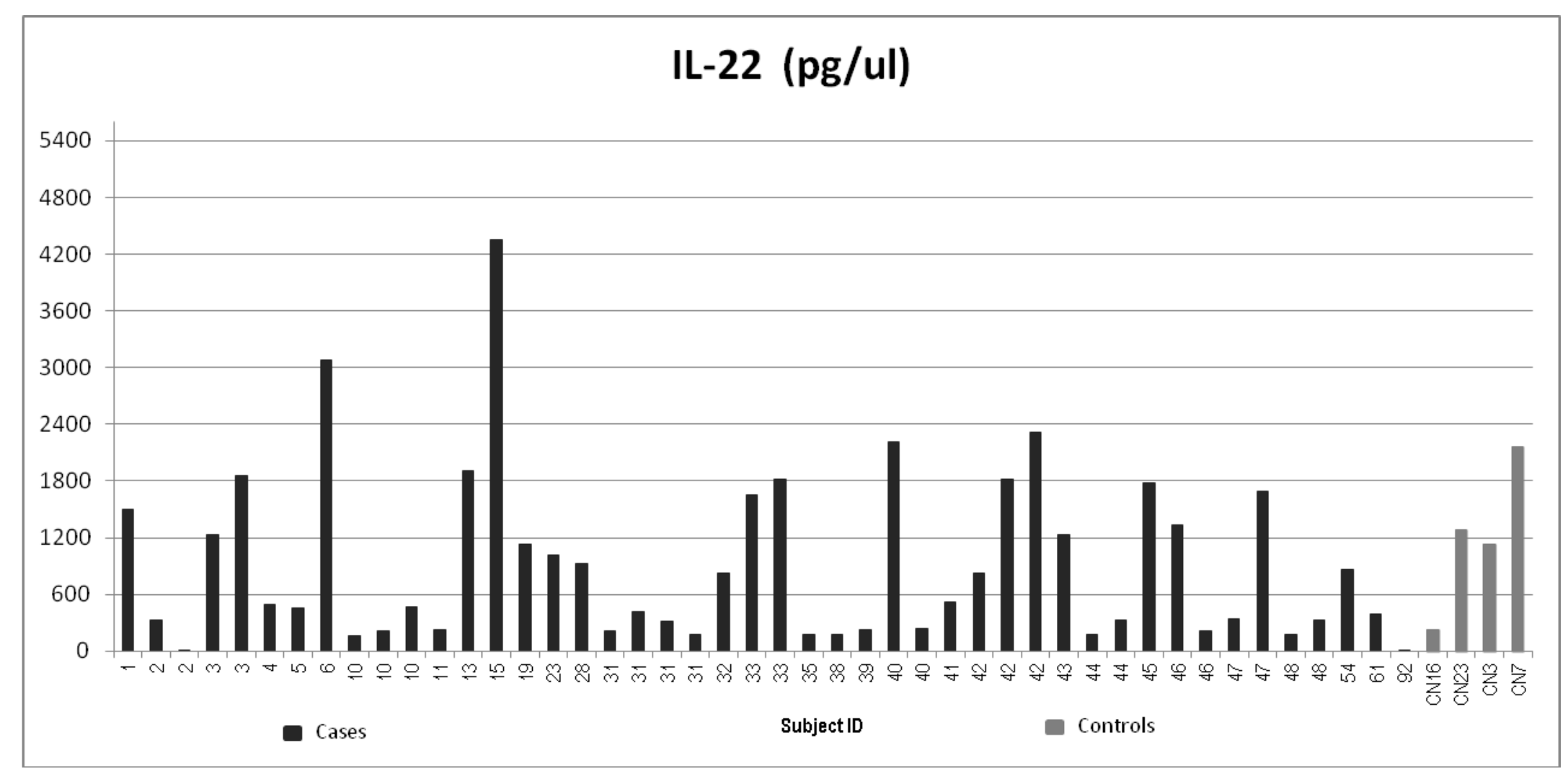




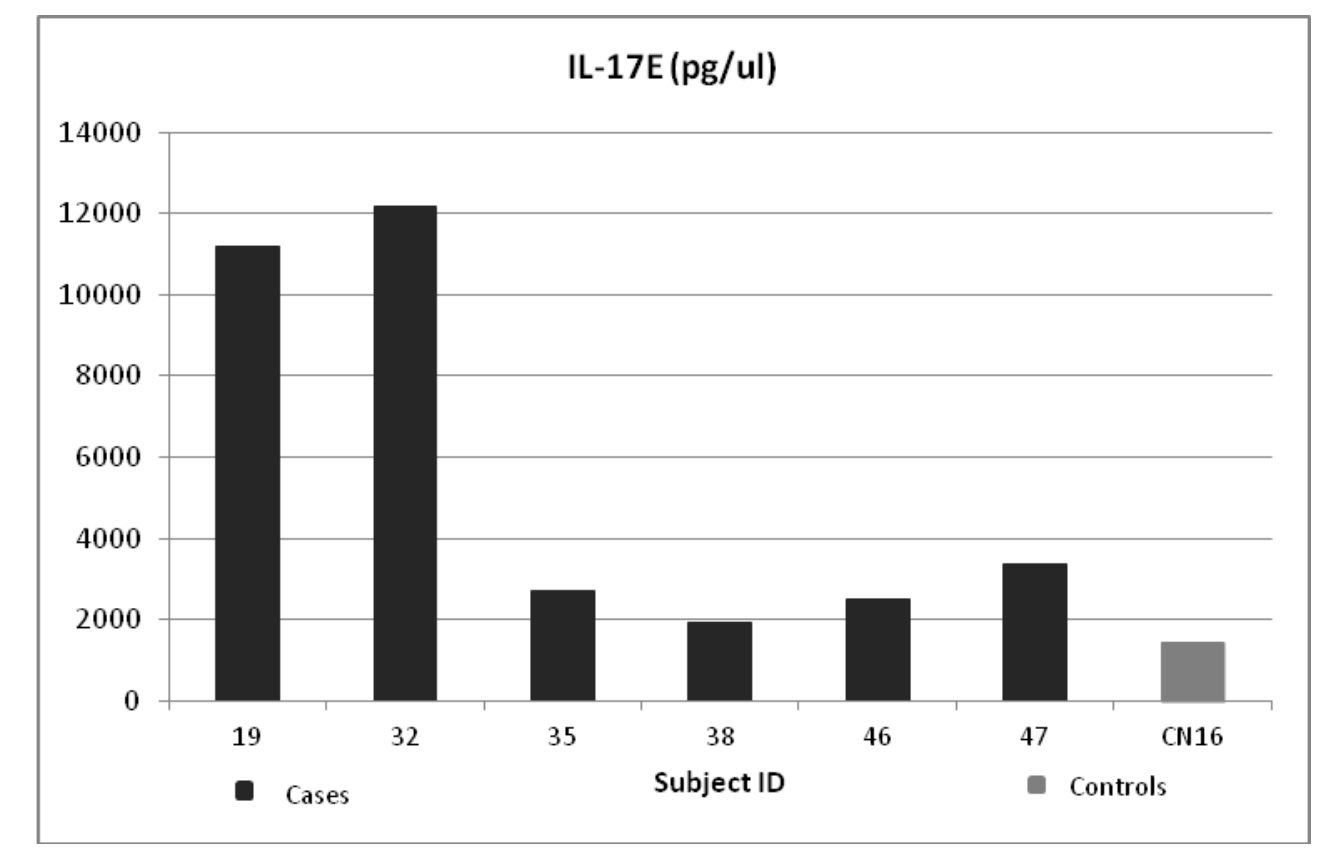

Figure 5_previously fig 7 


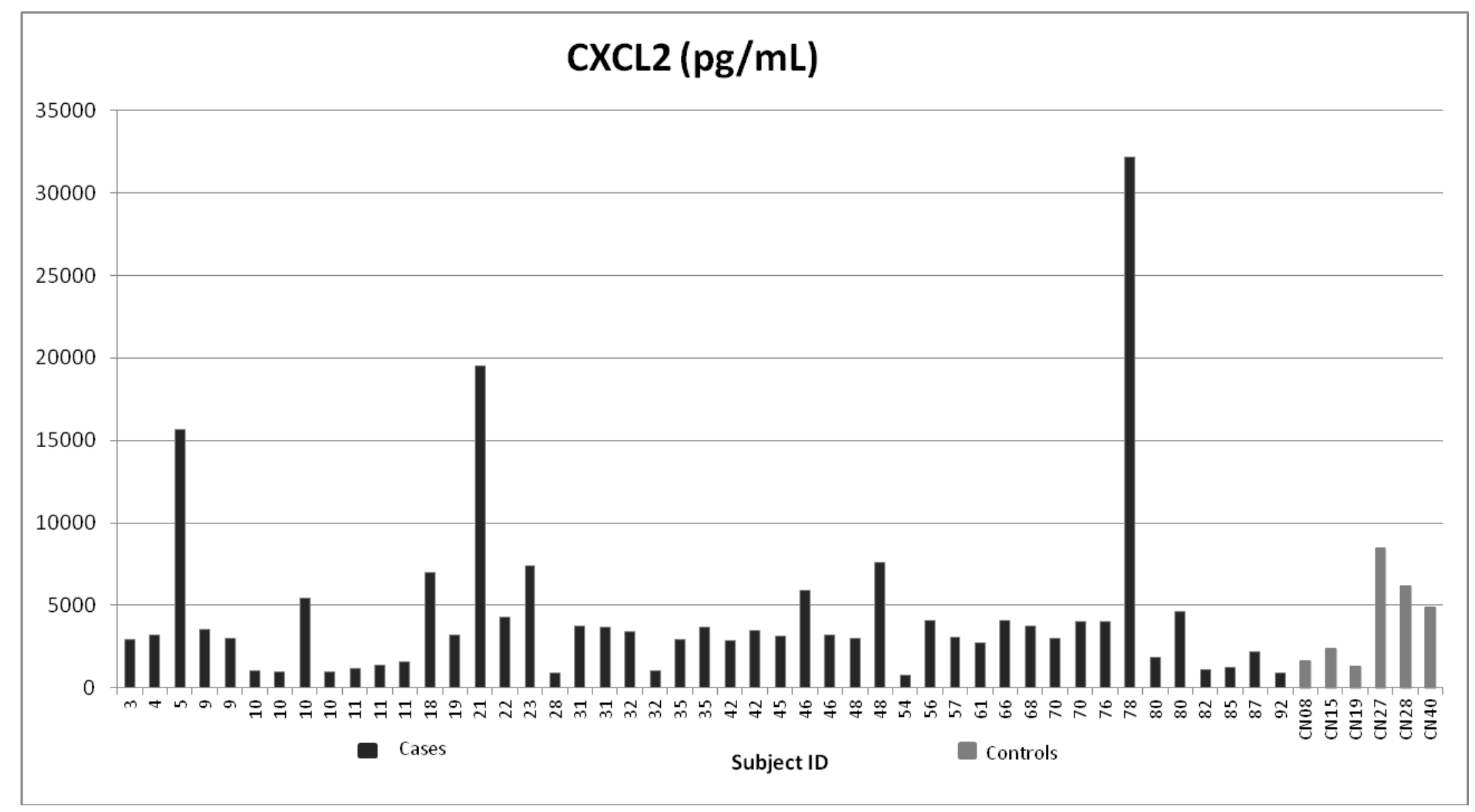




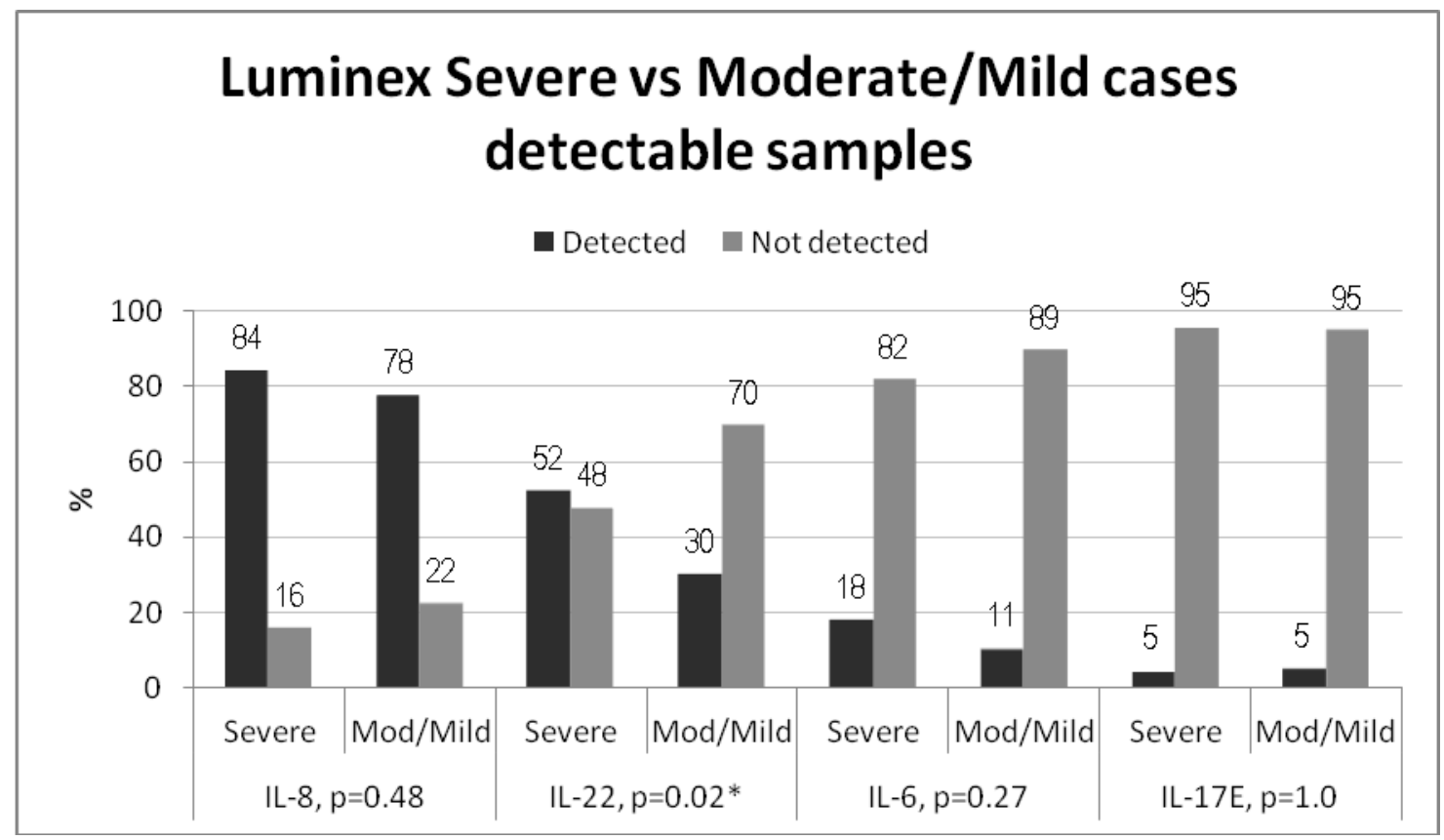

